\title{
THE PHYSICAL AND CHEMICAL EFFECTS OF C-SHOCKS IN MOLECULAR OUTFLOWS
}

\author{
G. PINEAU DES FORÊTS \\ DAEC, Observatoire de Paris \\ F-92195 Meudon Principal Cedex \\ France \\ D.R. FLOWER \\ Department of Physics, University of Durham \\ Durham DH1 3LE \\ $U K$ \\ AND \\ J.-P. CHIÈZE \\ DSM/DAPNIA/Service d'Astrophysique \\ CE-Saclay \\ F-91191 Gif-sur-Yvette Cedex \\ France
}

\begin{abstract}
The bow shocks which are observed in jets associated with lowmass star formation probably have C-shock characteristics over at least part of their extent. We show that ion-neutral streaming in C-type shocks can give rise to the release of significant amounts of silicon from the refractory cores of dust grains and hence to the formation of $\mathrm{SiO}$ in the gas phase. The computed profiles of $\mathrm{SiO}$ emission lines are found to be qualitatively similar to those observed in molecular outflow regions such as L1448. The question of the validity of the stationary-state assumption, adopted in all previous studies of C-shocks in the interstellar medium, is addressed. Timedependent calculations of the evolution of a $\mathrm{C}$-type shock propagating in low-density gas suggests that steady state is unlikely to be attained under the dynamical conditions in outflows.
\end{abstract}




\section{Introduction}

Observations of the jets associated with low-mass star formation, discussed in this volume, provide abundant evidence for the presence of bow shocks. The latter are believed to have J-shock characteristics at the apex and Cshock characteristics in the wake (Smith and Brand 1990). At the apex, molecular hydrogen is collisionally dissociated, whereas, in the wake, $\mathrm{H}_{2}$ is rovibrationally excited (Davis and Eisloeffel 1995; Gredel 1994, 1996).

Further evidence of the effects of shocks comes from observations of molecular lines in outflows, particularly of emission from rotationally excited states of $\mathrm{SiO}$ (Bachiller et al. 1991; Bachiller and Gomez-Gonzalez 1992; Martin-Pintado et al. 1992; Zhang et al. 1995). The SiO emission is found at velocities and positions which differ from those characterizing the ambient medium. In addition, the $\mathrm{SiO}: \mathrm{H}_{2}$ abundance ratio in the high velocity gas $\left(10^{-7}\right.$ to $\left.10^{-6}\right)$ is much higher than in the ambient medium (less than $10^{-11}$ ). Thus, the $\mathrm{SiO}$ emission appears to be intimately associated with dynamical activity and almost certainly with the presence of shocks.

The fraction of elemental silicon which is deduced from observations of the gas phase varies greatly amongst the different components of the interstellar medium. In diffuse clouds, from a few per cent to 70 per cent of the $\mathrm{Si}$ is observed to be in the gas phase (Sofia et al. 1994; Fitzpatrick 1996). In dark clouds, on the other hand, where most of the gas-phase silicon is expected to be in the form of $\mathrm{SiO}$ (Herbst et al. 1989), very low upper limits $\left(<3 \times 10^{-12}\right)$ have been established to the $\mathrm{SiO}: \mathrm{H}_{2}$ abundance ratio (Ziurys et al. 1989; Martin-Pintado et al. 1992). Indeed, $\mathrm{SiO}$ emission is observed only from dynamically active regions: molecular outflows, supernovae remnants and the Galactic centre. In quiescent clouds, silicon must be present in the solid phase, most probably in the form of refractory silicate grains or, possibly, in the ice mantles of the dust grains.

In this review, we shall address the questions of how the silicon is released from the solid phase and what is its subsequent fate. The discussion will centre around the role played by C-type shocks. In view of the short timescales associated with dynamical events in outflows, we also present the results of calculations of the temporal evolution of C-type shocks; all previous work has been based on the assumption of steady state.

\section{Non-thermal sputtering of grains by shocks in the interstellar medium}

In regions of high fractional ionization (the 'warm' or 'diffuse' interstellar medium), the neutral fluid is well coupled to the ions and thereby to the magnetic field. Under these conditions, shocks tend to be J-type and betatron acceleration of the (charged) grains occurs, owing to the compression 
of the gas and hence of the magnetic field. The charged grains orbit the magnetic field lines and acquire velocities, relative to neutral species, which are sufficient to give rise to sputtering (Draine and Salpeter 1979; Seab and Shull 1983; McKee et al. 1987; Jones et al. 1994, 1996). Shock speeds in the range $50 \lesssim v_{s} \lesssim 200 \mathrm{~km} \mathrm{~s}^{-1}$ yield impact energies, for helium atoms, for example, between about $50 \mathrm{eV}$ and $1 \mathrm{keV}$, which are sufficient to erode and even to destroy refractory grains. Grain-grain collisions are also important.

In regions of low fractional ionization, on the other hand, the collisional coupling between the ionized and neutral fluids is insufficient to maintain a common flow speed, and C-type shocks tend to develop. In 'low' velocity shocks $\left(10 \lesssim v_{s} \lesssim 50 \mathrm{~km} \mathrm{~s}^{-1}\right)$, the differential flow velocity gives rise to He atom impact energies, on charged grains, of less than $50 \mathrm{eV}$, sufficient to erode the grain mantles (Draine et al. 1983; Flower and Pineau des Forêts 1994). The effects of such shocks on the grain cores is more uncertain because of the lack of pertinent measurements of the sputtering yields in the low-energy domain: few experimental data are available below about $150 \mathrm{eV}$ (cf. Draine 1995).

\subsection{COMPUTED SPUTTERING YIELDS}

We have undertaken calculations of the sputtering yields for refractory materials, such as amorphous silica, $\mathrm{SiO}_{2}$ (Flower et al. 1996; Field et al. 1997), and, more recently, forsterite, $\mathrm{Mg}_{2} \mathrm{SiO}_{4}$. In Fig. 1 are presented the results of calculations of the sputtering yield of $\mathrm{Si}$ from amorphous $\mathrm{SiO}_{2}$ under impact by $\mathrm{He}^{+}$and heavier ions. The distinction between ionic and atomic impact is not significant in the context of the following discussion, because ions capture electrons as they approach the solid surface. The sputtering yield, $Y$, is defined as the ratio of the number of sputtered atoms of a given type to the total number of impacts and depends on both the velocity (and hence energy) and the angle, $\theta$, of impact. The results in Fig. 1 were obtained by means of the classical trajectory Monte Carlo code TRIM; for a description of the method, see Eckstein (1991). Up to $10^{7}$ trajectories were run for each value of $\theta$ and of the energy, $E$, of impact. The results were then averaged over the angle of incidence, relative to the normal to the surface, using the relation

$$
Y(E)=\int_{0}^{\pi / 2} Y(E, \theta) \sin \theta d \theta
$$

to obtain the results plotted in the Figure.

The very sharp decrease in the sputtering yields with decreasing impact velocity is evident from Fig. 1. We shall see below that the impact velocity is comparable with the shock velocity, $v_{s}$. It follows that, for $v_{s} \lesssim 50$ 


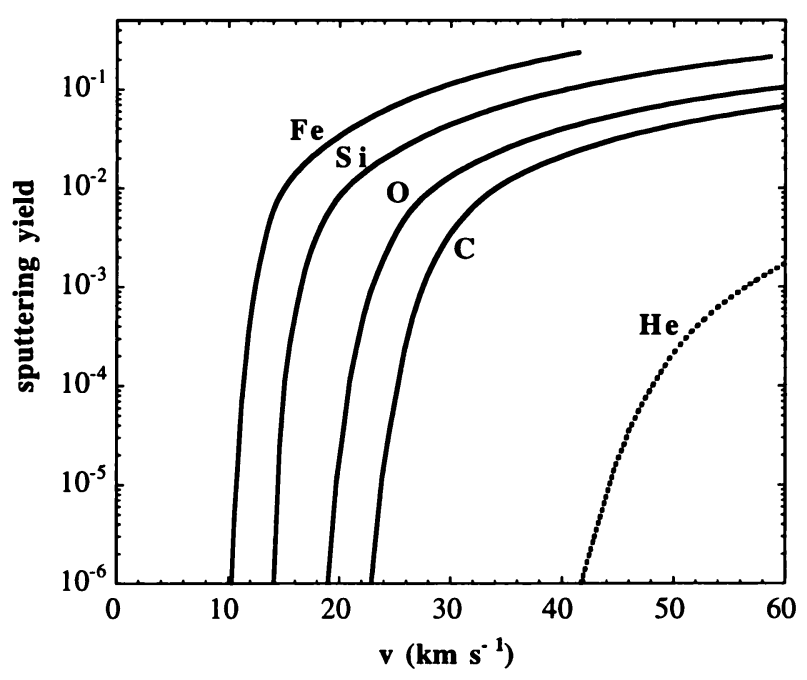

Figure 1. Sputtering yields of $\mathrm{Si}$ from amorphous $\mathrm{SiO}_{2}$, computed as functions of the velocity of incidence of helium and heavier projectile ions. The yields have been averaged over the angle of incidence.

$\mathrm{km} \mathrm{s}^{-1}$, the sputtering yield for He impact becomes much smaller than the yields for impact by heavier species. Indeed, the ratio of the sputtering yields more than compensates for the lower abundances of the heavy species, which, consequently, predominate at low shock speeds. Accordingly, in the calculations reported below, we have incorporated sputtering by the more abundant atomic and molecular species that are heavier than helium, adopting the sputtering yield calculated for the ion of nearest mass. This procedure receives its justification from the calculated sputtering yields, which show the impact energy (and hence the mass of the incident particle, at a given impact velocity) to be the determining factor.

\subsection{C-TYPE SHOCKS IN DARK MOLECULAR CLOUDS}

In dark clouds, the fractional ionization of the medium is so low that the collisional coupling between the neutral and the charged fluids is insufficient to prevent substantial differences developing in their flow speeds and kinetic temperatures. It is the charged fluid which couples directly to the magnetic field and receives 'advance notice' of the arrival of the shock wave if the Alfvén speed in the ionized fluid exceeds the shock speed. The charged fluid is then heated and accelerated prior to the neutral fluid (Mullan 1971; Draine 1980). In a C-type shock, the neutral fluid always flows supersonically relative to the shock front: radiative cooling prevents the sound speed, 

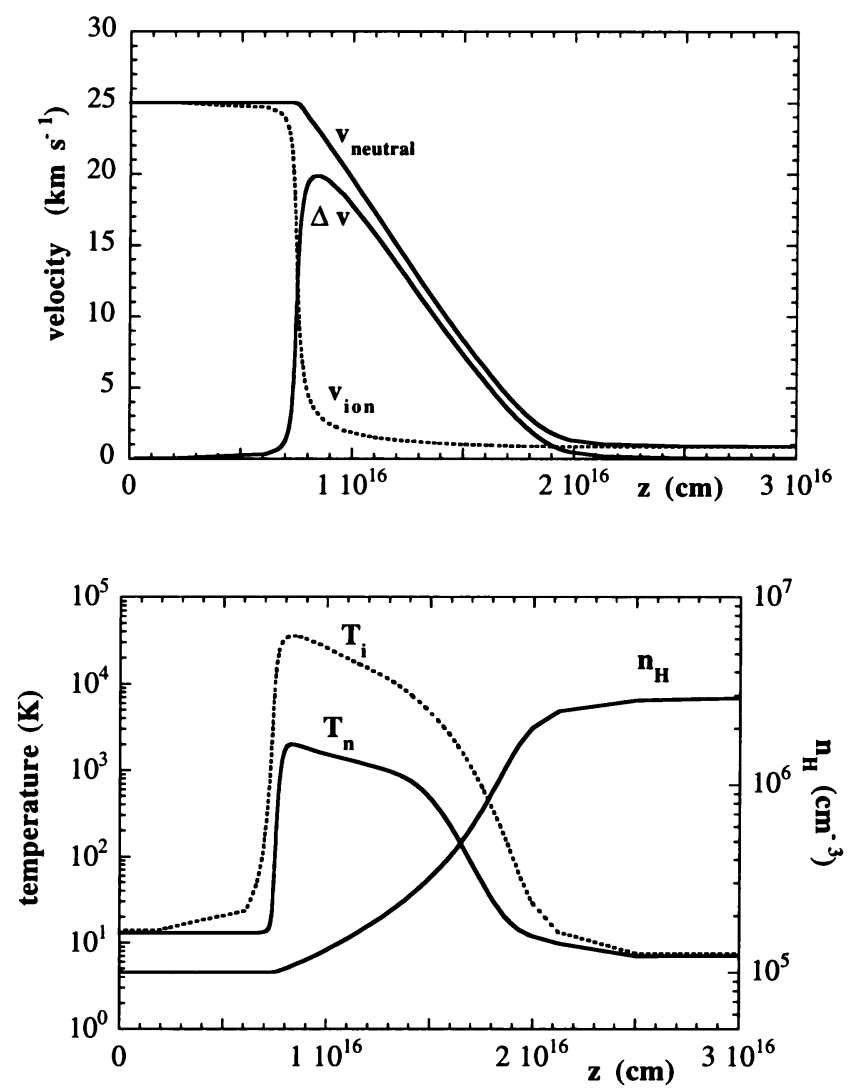

Figure 2. (a) Velocity and (b) temperature profiles for a shock wave of velocity $v_{s}=25$ $\mathrm{km} \mathrm{s}^{-1}$ propagating into a medium in which $n_{H}=10^{5} \mathrm{~cm}^{-3}$ and $B_{0}=200 \mu \mathrm{G}$. The ion-neutral drift speed is $\Delta v=v_{n}-v_{i}$ in the shock frame, in which the preshock gas flows at the shock speed, $v_{s}$. 'Preshock' is to the left and 'postshock' to the right in this Figure.

$c_{s}$, from attaining the local flow speed, $v_{n}$. (The appearance of a sonic point, $v_{n}=c_{s}$, in the flow is generally associated with a discontinuity.)

In Fig. 2, we show the stationary-state velocity and temperature profiles of a C-type shock propagating at $25 \mathrm{~km} \mathrm{~s}^{-1}$ into a medium of density $n_{H}=$ $n(H)+2 n\left(H_{2}\right)=10^{5} \mathrm{~cm}^{-3}$ where the magnetic induction is $B_{0}=200 \mu \mathrm{G}$, transverse to the flow direction. Compression of the neutral gas occurs over an extended distance, of the order of $10^{16} \mathrm{~cm}$. The difference $\Delta v$ between the flow speeds of the charged and neutral fluids may be seen to peak at a value not much smaller than the shock speed. The velocity of impact of the neutral particles on the charged grains is equal to the ion-neutral velocity difference. Each grain collides with many times its own mass of gas as it 
passes through the shock wave.

In the calculations reported below, the size distribution of the grains has been taken from Mathis et al. (1977). Individually, the grains are much more massive than the atomic and molecular ions, and, in a medium of low fractional ionization, their contribution to momentum transfer to the neutrals must be taken into account (Draine 1995; Flower and Pineau des Forêts 1995). In this context, it is necessary to establish the degree to which the grains are coupled to the magnetic field and hence flow at the same speed as the other charged particles.

The gyromagnetic frequency of a grain of mass $m_{g}$ and charge $Z e$ in a magnetic induction $B$ is given by

$$
\nu=\frac{B Z e}{2 \pi m_{g}},
$$

whereas the time, $\tau$, required for the grain to collide with its own mass of neutral gas is determined by

$$
\tau^{-1}=\frac{\rho_{n}}{m_{g}} \pi a^{2}\left|v_{i}-v_{n}\right|
$$

where $\rho_{n}$ is the mass density of the gas and $a$ is the radius of the grain. The grains are coupled to the magnetic field if $\nu>\tau^{-1}$, whence

$$
a(\mu m)<0.02(Z b)^{\frac{1}{2}}\left[\frac{\left|v_{i}-v_{n}\right|}{10 \mathrm{~km} \mathrm{~s}^{-1}}\right]^{-\frac{1}{2}}\left[\frac{n_{H}}{10^{4} \mathrm{~cm}^{-3}}\right]^{-\frac{1}{4}}
$$

and we have assumed that $B(\mu \mathrm{G})=b n_{H}^{\frac{1}{2}}$. In the preshock gas, $b \approx 1$, but the differential compression of the ions ensures that $b \gg 1$ in the shock. Furthermore, the magnitude $Z$ of the grain charge may be much larger than 1 in the shock (Caselli et al. 1997). It follows that most of the grains in the population that we adopt are well coupled to the magnetic field for the range of physical conditions that will be considered.

\subsection{NUMERICAL SIMULATIONS}

We shall present illustrative results for a model with the parameters specified above $\left(v_{s}=25 \mathrm{~km} \mathrm{~s}^{-1}, B_{0}=200 \mu \mathrm{G}, n_{H}=10^{5} \mathrm{~cm}^{-3}\right)$, incorporating about 100 gas-phase species and 800 chemical reactions. The grains are treated as being composed of a nucleus of amorphous silica surrounded by a mantle of ice containing up to 12 species, of which the most abundant are saturated hydrogenated molecules such as $\mathrm{H}_{2} \mathrm{O}$. More detailed information on the model is to be found in Schilke et al. (1997). 
It is essential to treat the chemistry, particularly the ion-neutral chemistry, in parallel with the dynamics. The gas is partially neutralized in the shock wave, owing to the enhanced rates of certain ion-neutral reactions which have activation energies. As a consequence, the dynamical structure of the shock wave is profoundly modified. Calculations reported in Section 3 below will serve to emphasize this point.

The mantles of the grains are rapidly removed in the shock wave, exposing the grain core. In the model presented here, a few per cent of the elemental silicon is then released from the grain nucleus by sputtering. This process is also rapid, occurring in the vicinity of the maximum of $\Delta v=\left|v_{i}-v_{n}\right|$. Once in the gas phase, the atomic silicon is oxidized, first to $\mathrm{SiO}$ and subsequently to $\mathrm{SiO}_{2}$, in the reactions

$$
\begin{gathered}
\mathrm{Si}+\mathrm{O}_{2} \longrightarrow \mathrm{SiO}+\mathrm{O} \\
\mathrm{Si}+\mathrm{OH} \longrightarrow \mathrm{SiO}+\mathrm{H} \\
\mathrm{SiO}+\mathrm{OH} \longrightarrow \mathrm{SiO}_{2}+\mathrm{H}
\end{gathered}
$$

The second stage of the oxidation process (formation of $\mathrm{SiO}_{2}$ from $\mathrm{SiO}$ ) is slower than the first but, nonetheless, it is effective owing to the enhanced abundance of $\mathrm{OH}$ in the shock-heated gas. $\mathrm{SiO}_{2}$ is the most abundant silicon-bearing species for a considerable distance into the postshock gas but is invisible, as it does not possess a permanent dipole moment. Eventually, $\mathrm{SiO}_{2}$ is removed from the gas phase by accretion on to the residual grain cores.

The flow time, $t$, of the neutrals is related to the distance, $z$, by the relation

$$
t=\int \frac{1}{v_{n}} d z
$$

Fig. 3 compares fractional abundances, expressed as functions of the flow time, calculated including and neglecting the re-accretion of gas-phase species on to grains. In the latter case, $\mathrm{SiO}_{2}$ is the most abundant $\mathrm{Si}$-bearing species for times comparable with the estimated lifetimes of molecular clouds $\left(10^{5}\right.$ $\mathrm{yr}$ ), when the fractional abundance of $\mathrm{SiO}$ is only of the order of $10^{-11}$. $\mathrm{SiO}_{2}$ is ultimately destroyed in reactions with ions, yielding $\mathrm{SiO}$. However, in the molecular outflows associated with low-mass star formation, the dynamical timescales $\left(10^{3}-10^{4} \mathrm{yr}\right)$ are too short for the oxidation of $\mathrm{SiO}$ to $\mathrm{SiO}_{2}$ to be completed, and so $\mathrm{Si}$ and $\mathrm{SiO}$ are likely to be the most abundant silicon-bearing species in the gas phase.

Once the physical and chemical structure of the shock wave has been determined, the profiles of the $\mathrm{SiO}$ emission lines can be calculated (Schilke et al. 1997). In Fig. 4, we compare the profiles of $\mathrm{SiO}$ rotational lines observed in the outflow L1448 (Bachiller et al. 1991) with the computations, 

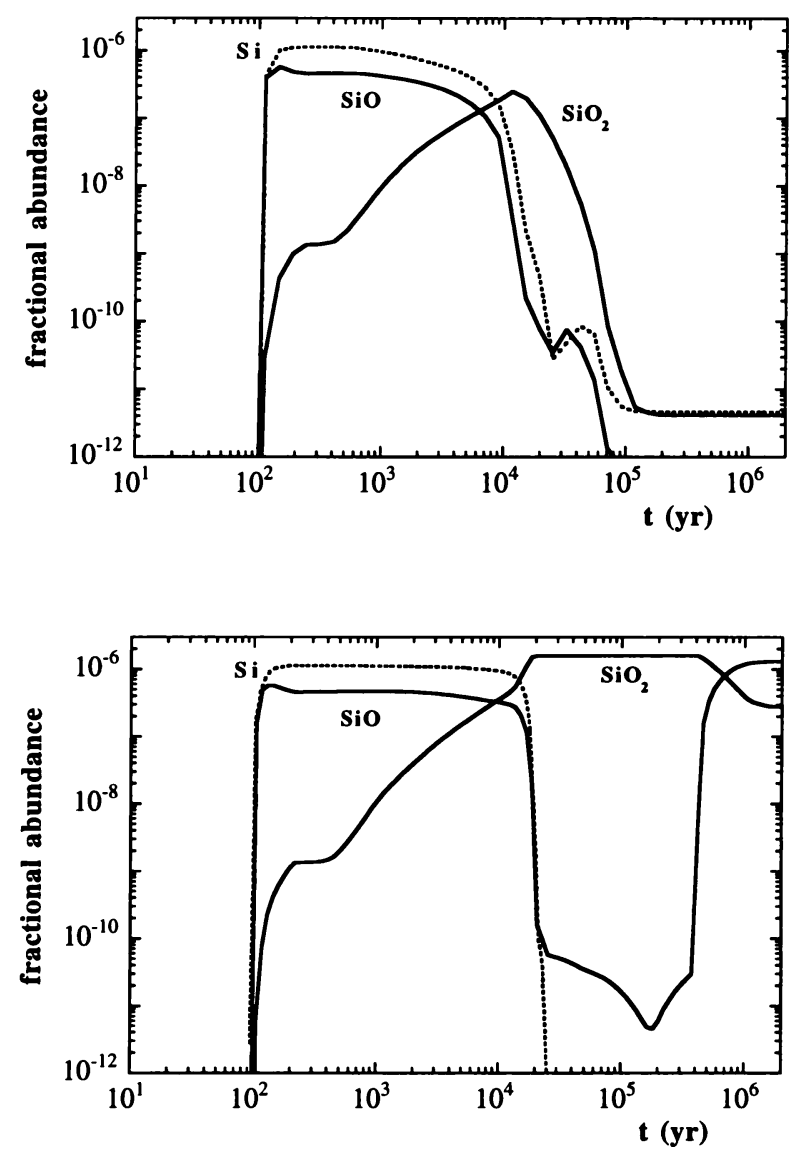

Figure 3. (a) Illustrating the release of $\mathrm{Si}$ into the gas phase and its subsequent oxidation to $\mathrm{SiO}$ and $\mathrm{SiO}_{2}$ prior to re-accretion by the grains. The independent variable is the flow time, $t$. (b) Neglecting re-accretion on to the grains. In this case, the decrease in the fractional abundance of $\mathrm{SiO}_{2}$ at large times is due to reactions with ions, which yield $\mathrm{SiO}$.

which relate to the blue-shifted components. It may be seen that the characteristic shapes of the lines are well reproduced by the model. Corrections for the beam filling factors have not been made, and so the absolute values of the observed and calculated brightness temperatures are not comparable. Analogous calculations are planned incorporating sputtering yields for more realistic grain materials (e.g. forsterite). Other species observed in outflows, such as CS and SO (see the review of Bachiller and Pérez Gutiérrez in this volume), will also be studied. Furthermore, we are currently investigating the rovibrational transitions of $\mathrm{H}_{2}$ arising from such shock waves. 

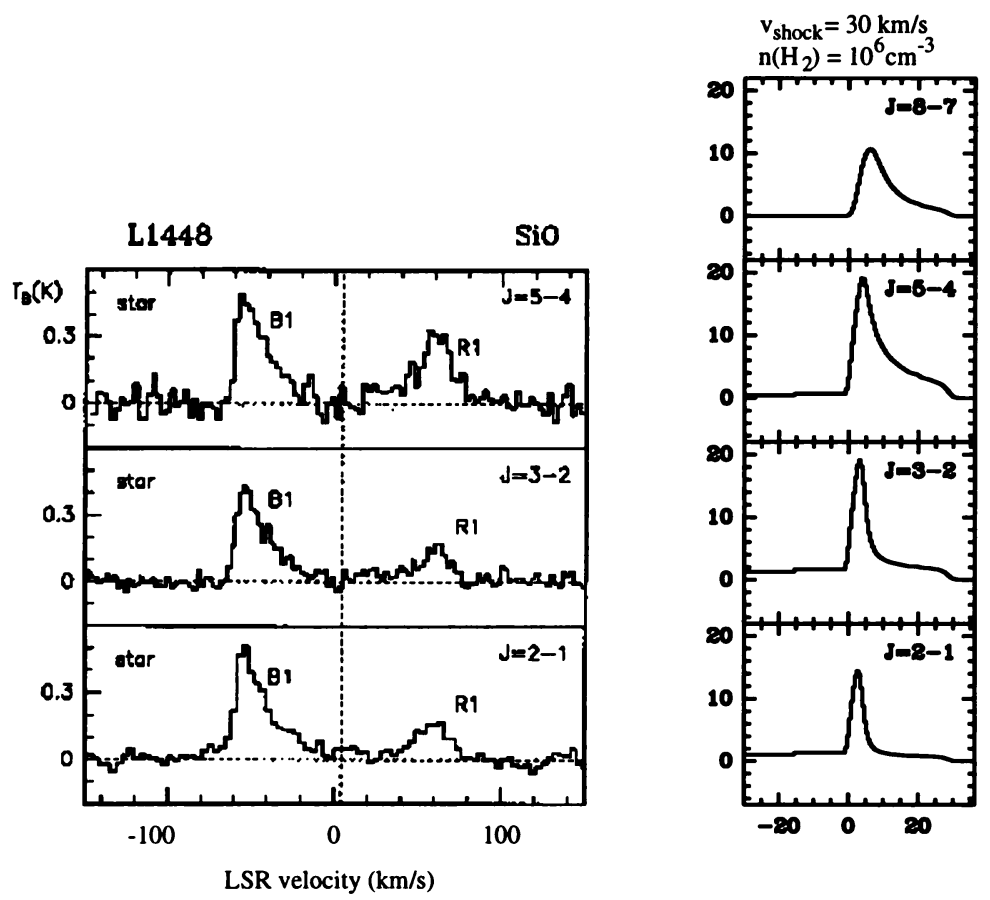

Figure 4. Observed and computed profiles of rotational transitions of $\mathrm{SiO}$; the calculations refer to the blue (B1) component. Corrections for beam filling factors have not been made, and so the absolute values of the brightness temperature, $T_{B}$, are not comparable.

\section{The temporal evolution from J- to C-type shocks}

Molecular outflows are examples of regions where major dynamical changes in the flow are likely to occur on timescales shorter than is required for the flow to reach a stationary state. Under these circumstances, a study of the propagation of shock waves should be based on the partial differential equations which describe the variation of the fluxes of number density, mass, momentum and energy of the charged and neutral fluids with position and time.

We have already mentioned in Section 2 the dynamical importance of the chemistry, specifically the ion-molecule chemistry; this is illustrated by the calculations in Fig. 5, which compares results obtained, at steady state, for a model in which $v_{s}=10 \mathrm{~km} \mathrm{~s}^{-1}, B_{0}=25 \mu \mathrm{G}$ and $n_{H}=10^{3} \mathrm{~cm}^{-3}$. In one calculation, the effects of chemical reactions were included, in the other, they were neglected. In the latter case, the fractional ionization of the gas is modified only by the differential compression of the ionized and neutral fluids by the shock wave and has the same value in the preshock and the 

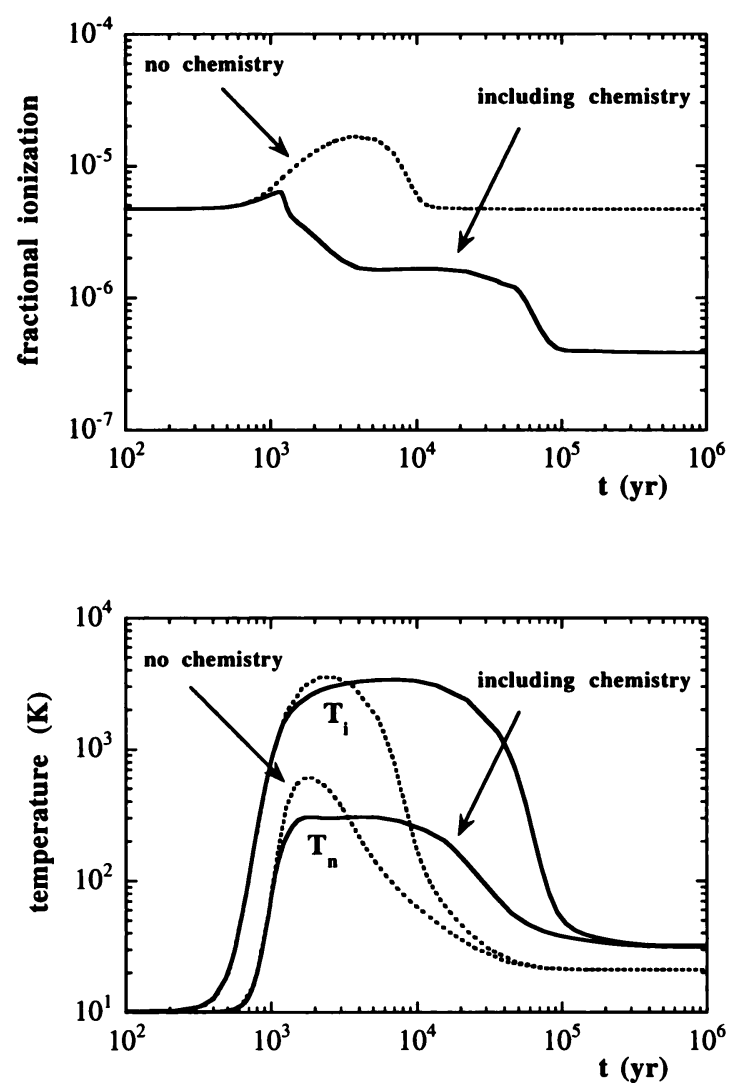

Figure 5. (a) Fractional ionization predicted by a model in which $v_{s}=10 \mathrm{~km} \mathrm{~s}^{-1}$, $n_{H}=10^{3} \mathrm{~cm}^{-3}$ and $B_{0}=25 \mu \mathrm{G}$; results are compared including and neglecting chemical reactions. (b) Corresponding temperature profiles of the ionized and neutral fluids.

postshock gas. The much lower degree of ionization, when chemical reactions are included, leads to weaker ion-neutral coupling, thereby enhancing the width of the shock wave and the time required to re-establish equilibrium, in the postshock gas. We note that the time required to attain the new equilibrium state is greater than or equal to the flow time through the shock wave, as is apparent from this Figure. The maximum value attained by the kinetic temperature of the neutral fluid is lower when chemical reactions are included. It is clear from Fig. 5 that the dynamics and the chemistry are intimately linked and have to be treated in parallel; this has been done in all the other calculations reported in this review.

A potential difficulty in treating the temporal evolution of shocks is the occurrence of sonic point(s) in the flow. The 'pseudo-viscosity' method of 
von Neumann and Richtmyer (see Richtmyer 1957) has been extensively used to address this problem. The technique involves artificially broadening the discontinuity so that it may be passed in a few integration steps without the necessity of impractically small step lengths. However, precautions have to be taken when collisional processes, in addition to those giving rise to viscosity, are involved. In the calculations reported below, this has been achieved by modifying the rate coefficients of all collisional processes (inelastic collisions, chemical reactions) by the ratio of the true to the pseudo-viscosity coefficient of the gas. A subsequent scaling procedure restores the correct time and distance scales to the computed variations in the extensive physical parameters; for further information, see Chièze et al. (1997).

Solving the time-dependent hydrodynamical equations is computer time consuming, and so the extent of the chemical network and number of chemical species must be limited. In the calculations that we shall now discuss, a network of 130 chemical reactions, involving 32 chemical species comprising the elements $\mathrm{H}, \mathrm{He}, \mathrm{C}, \mathrm{O}$ and $\mathrm{Fe}\left(\mathrm{Fe}^{+}\right.$is a representative heavy ion), was included alongside the hydrodynamical equations. There is then a total of 40 dependent variables per $z$-mesh which must be determined as functions of the independent variable, $t$. The step size used for the independent variable is adjusted automatically. Effects associated with the presence of dust grains have been neglected in these calculations.

In the model being considered, it has been assumed that a 'piston' acts continuously on the medium, driving a shock wave into the gas and imposing the postshock velocity. The shock wave, which is initially J-type, advances into the gas at a velocity which slightly exceeds that of the piston. As time progresses, the mass of the postshock material gradually increases until a steady state is finally attained. A comparison may then be made with the results of a stationary state calculation.

Fig. 6 shows the evolution of the temperature and density profiles of a shock wave whose parameters are those specified above. Even at an early time $\left(t=10^{2} \mathrm{yr}\right)$, the perturbation has travelled much further in the ionized fluid (at the ion Alfvén speed) than in the neutral fluid (at the shock speed). A discontinuity in the flow variables of the neutral gas clearly remains at $t=10^{3} \mathrm{yr}$. It is only much later $\left(t=510^{5} \mathrm{yr}\right)$, at a time which is comparable with the cooling time in the postshock gas (Fig. 5), that steady state is attained. At this point, the time-dependent profiles become indistinguishable from those generated by an independent, stationary-state calculation, affording at least a partial check on the validity of the results. The discontinuity in the neutral flow becomes weaker as time progresses and disappears completely when the width of the shock wave becomes equal to the distance required for the velocities of the charged and neutral fluids to recouple. 

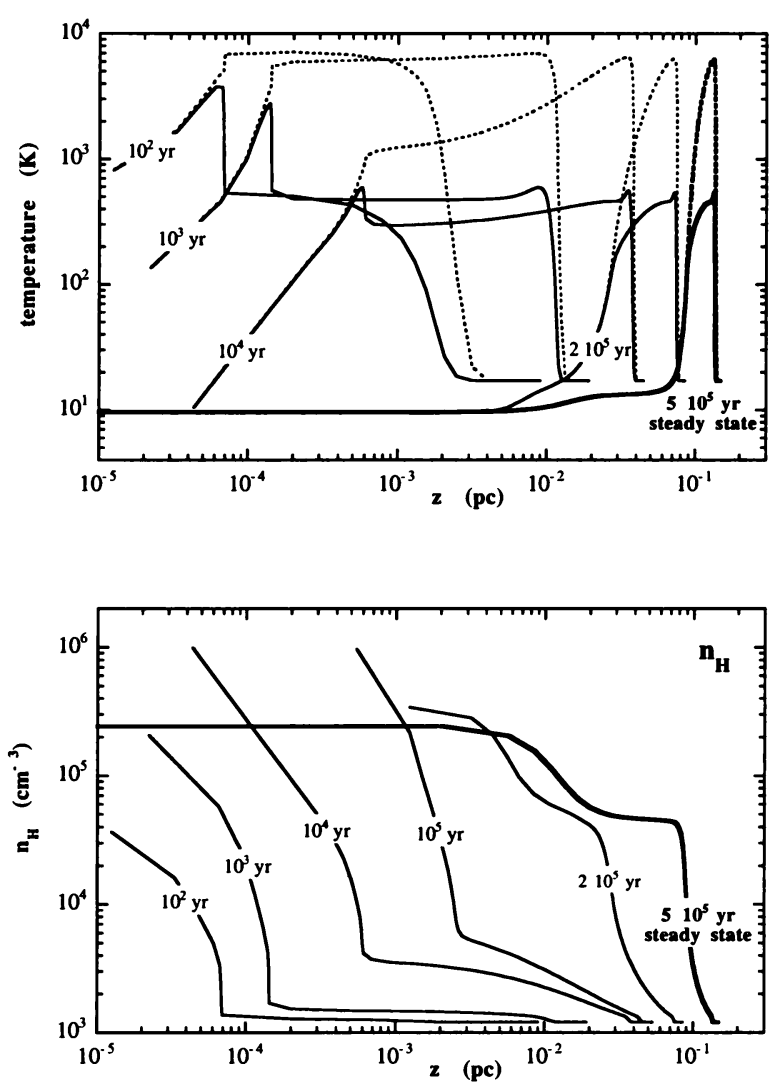

Figure 6. (a) Temperature and (b) density profiles as functions of position and time for the same model as in Fig. 5; chemical reactions are included. The shock wave advances from left to right, where a stationary state is finally attained.

In the context of the jets and outflows associated with low-mass star formation, an important point which emerges from Fig. 6 is that the time required to reach a stationary state is much greater than the dynamical timescales associated with such regions. Time-dependent calculations of, for example, the intensities of the emission lines of $\mathrm{H}_{2}$ and the process of grain erosion, then become necessary. However, we note that the preshock gas density in this model $\left(n_{H}=10^{3} \mathrm{~cm}^{-3}\right)$ is smaller than the densities that are believed to prevail in outflows, where the cooling times will be correspondingly shorter. Calculations for higher densities are planned but will prove very computer time consuming. 


\section{Concluding remarks}

We have shown that C-type shocks, with velocities in the range $20 \lesssim v_{s} \lesssim 50$ $\mathrm{km} \mathrm{s}^{-1}$, give rise to complete sputtering of the mantles and partial sputtering of the refractory cores of interstellar grains by heavy particle impact. Release of silicon into the gas phase is followed by its rapid oxidation to $\mathrm{SiO}$, which is observed in molecular outflows, and then more slowly to $\mathrm{SiO}_{2}$ (which is not observed: $\mathrm{SiO}_{2}$ has no permanent dipole moment). The computed profiles of the $\mathrm{SiO}$ emission lines are found to be qualitatively similar to those which are observed in outflows such as L1448.

Time-dependent calculations of shock propagation in a low-density $\left(n_{H}=\right.$ $10^{3} \mathrm{~cm}^{-3}$ ) molecular medium suggest that, in outflow regions, steady state may not be attained between the arrival of successive 'bullets' of outflowing material. At times significantly shorter than required to reach steady state, even a planar shock wave will have both C- and J-type characteristics, i.e. there will be an extended precursor region, followed by a quasidiscontinuous change in the flow variables. Calculations for higher preshock gas densities are necessary to confirm this result.

\section{Acknowledgements:}

We are grateful to the British Council and the Ministère des Affaires Etrangères for support under the 'Alliance' programme, contract no. 96026.

\section{References}

Bachiller, R., Martin-Pintado, J. and Fuente, A. (1991) $A \& A, 243$, L21.

Bachiller, R. and Gomez-Gonzalez, J. (1992) A\& A Rev., 3, 257.

Caselli, P., Hartquist, T.W. and Havnes, O. (1997), $A \mathcal{E} A$, in press.

Chièze, J.-P., Pineau des Forêts, G. and Flower, D.R. (1997) MNRAS, to be submitted.

Davis, C.J. and Eisloeffel, J. (1995) $A \mathscr{E} A$ 300, 851.

Draine, B.T. (1980) Ap.J. 241, 1021.

Draine, B.T. (1995) Ap. Sp. Sci. 233, 111.

Draine, B.T. and Salpeter, E.E. (1979) Ap.J. $231,438$.

Draine, B.T., Roberge, W.G. and Dalgarno, A. (1983) Ap.J. 264, 485.

Eckstein, W. (1991) Computer Simulation of Ion-Solid Interactions, Springer-Verlag, Berlin.

Field, D., May, P.W., Pineau des Forêts, G. and Flower, D.R. (1997) MNRAS, 285, 839.

Fitzpatrick, E.L. (1996) Ap.J. 473, L55.

Flower, D.R. and Pineau des Forêts, G. (1994) MNRAS 268, 724.

Flower, D.R. and Pineau des Forêts, G. (1995) MNRAS 275, 1049.

Flower, D.R., Pineau des Forêts, G., Field, D. and May, P.W. (1996) MNRAS 280, 447.

Gredel, R. (1994) $A \& \mathcal{G} A$ 292, 580.

Gredel, R. (1996) $A \& A$ 305, 582.

Herbst, E., Millar, T.J., Wlodek, S. And Bohme, D.K. (1989) A\& A 222, 205.

Jones, A.P., Tielens, A.G.G.M., Hollenbach, D.J. and McKee, C.F. (1994) Ap.J. 433, 797.

Jones, A.P., Tielens, A.G.G.M. and Hollenbach, D.J. (1996) Ap.J. 469, 740.

Martin-Pintado, J., Bachiller, R. and Fuente, A. (1992) $A \& A$ 254, 315.

Mathis, J.S., Rumpl, W. And Nordsieck, K.H. (1977) Ap.J. 217, 425. 
McKee, C.F., Hollenbach, D.J., Seab, C.G. and Tielens, A.G.G.M. (1987) Ap.J. 318, 674.

Mullan, D.J. (1971) MNRAS 153, 145.

Richtmyer, R.D. (1957) Difference methods for initial-value problems, Interscience, New York.

Schilke, P., Walmsley, C.M., Pineau des Forêts, G. and Flower, D.R. (1997) $A \mathscr{E} A$, in press.

Seab, C.G. and Shull, J.M. (1983) Ap.J. 275, 652.

Smith, M.D. and Brand, P.W.J.L. (1990) MNRAS 245, 108.

Sofia, U.J., Cardelli, J.A. and Savage, B.D. (1994) Ap.J. 430, 650.

Zhang, Q., Ho, P.T.P., Wright, M.C.H. and Wilner, D.J. (1995) Ap. J. 451, L71.

Ziurys, L.M., Friberg, P. and Irvine, W.M. (1989) Ap.J. 343, 201. 